\title{
DEFICIENT SUBSETS IN LOCALLY CONVEX SPACES
}

\author{
DOUGLAS W. CURTIS
}

1. Introduction. A closed subset of an infinite-dimensional linear topological space has topological infinite deficiency if there exists a space homeomorphism taking it in to a closed subspace with infinite codimension. A closed subset is said to have $\omega$-deficiency if there exist space homeomorphisms taking it into closed subspaces of every finite codimension. For the class of locally convex Hausdorff spaces, the former implies the latter.

Klee [5], [6] has shown that compact sets in infinite-dimensional Banach spaces have $\omega$-deficiency (in separable infinite-dimensional Hilbert space, topological infinite deficiency). We generalize this as follows: in normed spaces which have the property that no barrel is totally bounded, closed sets which are countable unions of totally bounded sets have $\omega$-deficiency. A principal application of the deficiency conditions is to the problem of extending homeomorphisms.

Using the natural imbedding of the Fréchet space $s$ (the countable infinite product of lines) in the Hilbert cube, Anderson [1], [2] characterizes topological infinite deficiency in separable infinite-dimensional Fréchet spaces as Property $Z$, a global and local homotopy condition on the complement of the set. Here, an alternative formulation of Property $Z$ (homotopy deficiency) is given, and it is shown that $\omega$-deficiency always implies homotopy deficiency. In the opposite direction, one may ask for conditions under which Property $Z$ implies $\omega$-deficiency, and $\omega$-deficiency implies topological infinite deficiency. At present, Anderson's theorem is apparently the strongest statement available.

The work presented here forms a part of the author's dissertation, written under the direction of Professor D. E. Sanderson at Iowa State University.

2. Preliminary lemmas. For proofs or methods of proof of the following, refer to Kelley and Namioka [4].

Lemma 2.1. Let $M$ be a finite-dimensional subspace of a locally convex Hausdorff linear space, and let $P$ be a closed subspace with $M \cap P=\theta$. Then $M$ has a closed complement $N$ with $N \supset P$.

Lemma 2.2. Let $X=M \oplus N$ be a decomposition of a linear topological

Presented to the Society, February 9, 1968 under the title Deficiency in infinitedimensional spaces; received by the editors July 5, 1968 . 
space, with $M$ finite-dimensional and $N$ closed. Then the projections onto $M$ and $N$ are continuous.

LEMMA 2.3. Every totally bounded subset of an infinite-dimensional Hausdorff linear space is nowhere dense.

LEMMA 2.4. The closed convex circled extension of a totally bounded subset of a locally convex linear space is totally bounded.

It follows from Lemma 2.1 that in infinite-dimensional locally convex Hausdorff spaces, subsets with topological infinite deficiency have $\omega$-deficiency. It is also true that such spaces contain closed infinite-dimensional subspaces with infinite codimension. The given conditions guarantee the existence of biorthogonal sequences $\left\{x_{i}, f_{i}\right\}$ of points and continuous linear functionals [7]. For any such sequence, the closed span of $\left\{x_{1}, x_{3}, \ldots\right\}$ is a subspace of the desired type.

3. $\omega$-deficiency. A barrel is a closed convex circled set which is radial at $\theta$. A locally convex space with the property that each barrel is a neighborhood of $\theta$ is said to be barrelled. Every space of the second category, hence every Fréchet space, is barrelled.

Lemma 3.1. For a locally convex linear metric space $X$, the following are equivalent:

(1) No barrel is totally bounded.

(2) No set radial at $\theta$ is totally bounded.

(3) $X$ is not the countable union of totally bounded sets.

Proof. By Lemma 2.4, (1) implies (2). Suppose $X=U B_{i}$ is the countable union of totally bounded sets; we may assume each $B_{i}$ is circled. With $N(r)=\{x: d(x, \theta)<r\}$, let $B=\mathrm{U}\left(B_{i} \cap N(1 / i)\right)$. Then $B$ is a totally bounded set radial at $\theta$ and (2) implies (3). Obviously (3) implies (1).

A locally convex linear metric space satisfying the above conditions will be called a $Q$-space. Clearly, a $Q$-space must have uncountable linear-dimension. By Lemma 2.3, every infinite-dimensional barrelled linear metric space is a $Q$-space. Let $X \subset l^{2}$ be the linear subspace spanned by $\left\{\left(y_{k}\right): y_{2 i-1} y_{2 i}=0, y_{2 i-1}+y_{2 i}=1 / i, i=1,2, \cdots\right\}$. Then $X$ is an uncountable-linear-dimensional inner-product space which is not a $Q$-space. However, we do have the following

THEOREM 3.2. Every nonseparable normed linear space is a $Q$-space.

Proof. Let $X$ be a nonseparable normed linear space, and let $M$ be a subset of the unit sphere, maximal with respect to the condition 
that $\|x-y\| \geqq \frac{1}{2}$ for $x, y \in M, x \neq y$. By Riesz's Lemma, $M$ must be uncountable. Let $R$ be any set radial at $\theta$. Then for some $\alpha>0$, there is an infinite subset $M_{\alpha}$ of $M$ such that $\alpha M_{\alpha} \subset R$. Obviously $\alpha M_{\alpha}$, and therefore $R$, cannot be totally bounded.

By a $\sigma$-totally bounded set we mean a countable union of totally bounded sets. The proof of the following fundamental lemma is essentially due to Klee [5].

Lemma 3.3. Let $A$ be a $\sigma$-totally bounded subset of a $Q$-space $X$. Then there is a line $L$ in $X$, no translate of which intersects the convex extension $\langle A\rangle$ more than once.

Proof. We may suppose $A=\bigcup A_{i}, A_{i} \subset A_{i+1}$, each $A_{i}$ totally bounded. By Lemma 2.4 each $\left\langle A_{i}\right\rangle$ is totally bounded. Since $\langle A\rangle$ $=\mathrm{U}\left\langle A_{i}\right\rangle,\langle A\rangle-\langle A\rangle$ is $\sigma$-totally bounded. For some $u \in X \backslash \theta$, the line $L=\{t u:-\infty<t<\infty\}$ intersects $\langle A\rangle-\langle A\rangle$ only at $\theta$. Otherwise, $\langle A\rangle-\langle A\rangle$ is radial at $\theta$, and $X$ is $\sigma$-totally bounded. Thus no translate of $L$ intersects $\langle A\rangle$ more than once.

THEOREM 3.4. Let $A$ be a closed totally bounded (resp. $\sigma$-totally bounded) subset of a $Q$-space (resp. normed $Q$-space) $X$. Then $A$ has $\omega$-deficiency in $X$.

Proof. Let $L=\{t u:-\infty<t<\infty\}$ be the line of Lemma 3.3, and let $H$ be a closed complementary hyperplane. Let $\tau: X \rightarrow H$ be the (continuous) projection onto $H$. Then $\tau \mid\langle A\rangle$ is $1-1$. If $A$ is totally bounded, $\tau \mid A$ is a homeomorphism onto $\tau(A)$, which is closed. The homeomorphism $(\tau \mid A)^{-1}$ defines a map $m_{A}: \tau(A) \rightarrow(-\infty, \infty)$ which can be extended to a map $m$ on $H$. Then $h+t u \rightarrow h+(t-m(h)) u$ defines a homeomorphism of $X$, extending $\tau \mid A$. Since $\tau(A)$ is totally bounded, a simple inductive argument shows that $A$ has $\omega$-deficiency.

Suppose $X$ is normed; assume $\|u\|=1$. For each $h \in H \backslash \theta$, consider the semicircle $S_{h}$ :

$$
S_{h}=\left\{t\|h\| u+r h: t^{2}+r^{2}=1, r \geqq 0\right\} .
$$

Each point of $X \backslash L$ lies on exactly one semicircle. Assuming $\theta \in A$, we have $A \subset(X \backslash L) \cup \theta$, and no semicircle intersects $A$ more than once (otherwise $\tau \mid\langle A\rangle$ is not 1-1). Let $S[A]$ denote the subfamily of semicircles that intersect $A$. Define a bijection $G_{A}: \cup S[A] \cup \theta \rightarrow \cup S[A] \cup \theta$ as follows: $G_{A}(\theta)=\theta$, and for $S_{h} \in S[A], G_{A} \mid S_{h}$ is fixed on the endpoints (in $L$ ), takes $S_{h} \cap A$ onto $h=S_{h} \cap H$, and is extended piecewise linearly (with respect to the $t$-coordinate) on $S_{k}$.

We show that $G_{A} \mid A$ is a homeomorphism and $G_{A}(A)$ is closed. Obviously $G_{A} \mid A$ is continuous. Suppose $G_{A}^{-1} \mid G_{A}(A)$ is not continuous. 
Then there exists a sequence $G_{A}\left(a_{n}\right) \rightarrow G_{A}(a), a_{n}, a \in A$, such that no subsequence of $a_{n}$ converges to $a$. With $a_{n}=t_{n}\left\|h_{n}\right\| u+r_{n} h_{n}$ and $a$ $=t\|h\| u+r h$, we have $G_{A}\left(a_{n}\right)=h_{n} \rightarrow h=G_{A}(a)$. Since there is some subsequence $a_{n^{\prime}}$ for which $t_{n^{\prime}}$ and $r_{n^{\prime}}$ converge, we have $a_{n^{\prime}} \rightarrow a^{\prime} \in A$, hence $G_{A}\left(a_{n^{\prime}}\right) \rightarrow G_{A}\left(a^{\prime}\right), G_{A}(a)=G_{A}\left(a^{\prime}\right), a=a^{\prime}$, and $a_{n^{\prime}} \rightarrow a$, contrary to hypothesis.

To see that $G_{A}(A)$ is closed, let $G_{A}\left(a_{n}\right) \rightarrow h \in H$. Then as before, $h_{n} \rightarrow h$, there is some subsequence $a_{n^{\prime}}$ such that $t_{n^{\prime}}$ and $r_{n^{\prime}}$ converge, thus $a_{n^{\prime}} \rightarrow a^{\prime} \in A, G_{A}\left(a_{n^{\prime}}\right) \rightarrow G_{A}\left(a^{\prime}\right)=h \in G_{A}(A)$, and $G_{A}(A)$ is closed.

The homeomorphism $G_{A}^{-1} \mid G_{A}(A)$ defines a map $m_{A}: G_{A}(A) \backslash \theta$ $\rightarrow(-1,1)$, with $m_{A}\left(G_{A}(t\|h\| u+r h)\right)=t$. Since $A$ and $G_{A}(A)$ are closed, and $L \backslash \theta$ does not intersect $A, m_{A}$ can be extended to a map $m: H \backslash \theta \rightarrow(-1,1)$ in such a way that, for every integer $N, m$ maps $\{h: 1 / N<\|h\|<N\}$ into a closed subinterval of $(-1,1)$. This extension may be used in the obvious manner to define a homeomorphism $G: X \rightarrow X$, extending $G_{A}$. Again an inductive argument completes the proof, provided $G_{A}(A)$ is $\sigma$-totally bounded. For this purpose we require the following lemma. The canonical notation is used: $y$ $=t\|h\| u+r h, y^{\prime}=t^{\prime}\left\|h^{\prime}\right\| u+r^{\prime} h^{\prime}$.

Lемма 3.5. For every $\epsilon>0, N>0$ there exists $\delta>0$ such that if $\left\|y-y^{\prime}\right\|<\delta, r>1 / N$, and $\left\|h^{\prime}\right\|<N$, then $\left\|h-h^{\prime}\right\|<\epsilon$.

Proof. We have

$$
\begin{aligned}
\left\|h-h^{\prime}\right\| & =(1 / r)\left\|\left(r h-r^{\prime} h^{\prime}\right)+\left(r^{\prime}-r\right) h^{\prime}\right\| \\
& \leqq(1 / r)\left(\left\|r h-r^{\prime} h^{\prime}\right\|+\left|r^{\prime}-r\right|\left\|h^{\prime}\right\|\right) .
\end{aligned}
$$

Since $\left|t\|h\|-t^{\prime}\left\|h^{\prime}\right\|\right| \rightarrow 0$ and $\left\|r h-r^{\prime} h^{\prime}\right\| \rightarrow 0$ as $\left\|y-y^{\prime}\right\| \rightarrow 0$, and since $\left|r\|h\|-r^{\prime}\left\|h^{\prime}\right\|\right| \leqq\left\|r h-r^{\prime} h^{\prime}\right\|$, it follows that $t\|h\| \rightarrow t^{\prime}\left\|h^{\prime}\right\|$ and $r\|h\|$ $\rightarrow r^{\prime}\left\|h^{\prime}\right\|$. Then $r^{2}+t^{2}=\left(r^{\prime}\right)^{2}+\left(t^{\prime}\right)^{2}=1$ implies that $r^{\prime} \rightarrow r$. Thus $\left\|h-h^{\prime}\right\| \rightarrow 0$ as $\left\|y-y^{\prime}\right\| \rightarrow 0$, subject to the given restrictions.

Clearly, Lemma 3.5 shows that the "projection" of $X \backslash L$ onto $H$ along semicircles takes totally bounded sets onto $\sigma$-totally bounded sets, and it follows that $G_{A}(A)$ is $\sigma$-totally bounded.

4. Homogeneity. Klee [6] has given a method for the extension, in certain situations, of homeomorphisms between deficient subsets of a normed space. The key lemma (which we state here (Lemma 4.1) in the form applicable to $\omega$-deficiency) is based on Dugundji's theorem that locally convex Hausdorff spaces are absolute extensors for the class of metrizable spaces.

Lemma 4.1. Let $X=M \oplus N$ be a decomposition of a locally convex linear metric space, with $M$ finite-dimensional and $N$ closed. Let 
$h: A \rightarrow B$ be a homeomorphism of a closed subset of $M$ onto a closed subset of $N$. Then there is an isotopy $\left\{\Lambda_{t}: t \in I\right\}$ of $X$ onto itself, such that $\left\{\Lambda_{t}^{-1}: t \in I\right\}$ is also an isotopy, with $\Lambda_{1}=\mathrm{id}$ and $\Lambda_{0} \mid A=h$.

For the application of Klee's method to locally compact subsets, the following lemma (proof suggested by D. E. Sanderson) is required:

LEMMA 4.2. Every finite-dimensional locally compact separable metric space $A$ can be imbedded as a closed subset of a Euclidean space.

Proof. The one-point compactification $A^{*}$ is finite-dimensional metrizable, and can be imbedded as a (closed) subset of $S^{n}$ for some $n$. Deleting the compactifying point $\omega$, we have $A=A^{*} \backslash \omega$ imbedded as a closed subset of $S^{n} \backslash \omega \sim R^{n}$.

Theorem 4.3. Let $X$ be a normed $Q$-space and let $h$ be a homeomorphism between closed finite-dimensional locally compact separable subsets of $X$. Then $h$ can be extended to a stable homeomorphism $H$ of $X$.

Proof. Let $h: A \rightarrow B$. By Theorem 3.4, $A \cup B$ has $\omega$-deficiency in $X$. Thus there exist a decomposition $X=T \oplus R \oplus N$, where $\operatorname{dim} T=1$, $R$ is a finite-dimensional subspace containing a closed copy $A^{\prime}$ of $A$, and $N$ is closed; and a homeomorphism $F$ of $X$ such that $F(A \cup B)$ $\subset N$. Applying Lemma 4.1 twice on $R \oplus N$ (first extending any homeomorphism of $F(A)$ onto $A^{\prime}$, then extending the appropriate homeomorphism of $A^{\prime}$ onto $F(B)$ ), we obtain an isotopy $\left\{\Lambda_{t}\right\}$ of $R \oplus N$, such that $\left\{\Lambda_{t}^{-1}\right\}$ is also an isotopy, with $\Lambda_{1}=$ id and $F^{-1} \Lambda_{0} F \mid A=h$. Define a homeomorphism $G: X \rightarrow X$ as follows:

$$
\begin{aligned}
G(t+r+n) & =t+\Lambda_{t}(r+n), & & 0 \leqq t \leqq 1 \\
& =t+\Lambda_{0}(r+n), & & t \leqq 0 \\
& =t+r+n, & & t \geqq 1 .
\end{aligned}
$$

Then $H=F^{-1} G F$ is a stable extension of $h$. (Note that for compact sets, the norm requirement on $X$ is unnecessary.)

5. Homotopy deficiency. $E^{n+1}$ will denote a closed $(n+1)$-cell, with $\operatorname{Bd}\left(E^{n+1}\right)=S^{n}$.

Definition. A closed nowhere dense subset $A$ of a topological space $X$ has homotopy deficiency in $X$ if, for every map $f: E^{n+1} \rightarrow X$ with $f\left(S^{n}\right) \cap A=\varnothing$, and every open cover $\alpha$ of $X$, there exists a map $g: E^{n+1} \rightarrow X$ such that $f\left|S^{n}=g\right| S^{n}, f$ and $g$ are $\alpha$-near, and $g\left(E^{n+1}\right) \cap A$ $=\varnothing$. 
For linear topological spaces the $\alpha$-near requirement of the above definition assumes the natural form $f(x)-g(x) \in V$ for all $x$ ( $V$ an arbitrary neighborhood of $\theta$ ). For metric spaces it becomes $d(f(x), g(x))<\epsilon$ for all $x$ (arbitrary $\epsilon>0$ ). A standard argument involving uniform convergence is available to show that, in complete metric spaces, closed sets which are countable unions of homotopically deficient sets are homotopically deficient.

THEOREM 5.1. Every $\omega$-deficient subset $A$ of a linear topological space $X$ is homotopically deficient.

Proof. Obviously $A$ is closed nowhere dense. Let $f: E^{n+1} \rightarrow X$ with $f\left(S^{n}\right) \cap A=\varnothing$, and let $V$ be a neighborhood of $\theta$. There exist a decomposition $X=L_{1} \oplus L_{2}$, with $L_{1}$ closed and $\operatorname{dim} L_{2}=n+2$, and a homeomorphism $h$ of $X$ with $h(A) \subset L_{1}$. By compactness there exists a neighborhood $W$ of $\theta$ such that, for $g: E^{n+1} \rightarrow X$, $(h f-h g)\left(E^{n+1}\right) \subset W$ implies $(f-g)\left(E^{n+1}\right) \subset V$. Thus we may assume that $A \subset L_{1}$. Let $\tau_{i}$ be the projection onto $L_{i}$ (continuous by Lemma 2.2), and consider $f$ $=\left(f_{1}, f_{2}\right)$, where $f_{i}=\tau_{i} f$. We construct $g=\left(g_{1}, g_{2}\right)$ with $f_{1}=g_{1}, f_{2} \mid S^{n}$ $=g_{2} \mid S^{n}$. If $L_{2}$ is non-Hausdorff, an indiscrete subspace can be factored out, and $g_{2}$ can then be constructed in a suitable manner. Otherwise, let \|\| be a norm on $L_{2}$ and let $N(r)=\left\{y \in L_{2}:\|y\|<r\right\}$. Choose $\epsilon>0$ such that $N(\epsilon) \subset V$. There exists $0<\delta<\epsilon / 2$ such that $S^{n} \cap f_{1}^{-1}(A)$ $\cap f_{2}^{-1}(N(\delta))=\varnothing$. With $C=f_{1}^{-1}(A)$, consider the restriction $f_{2} \mid C: C$ $\rightarrow L_{2}$. If $\theta \notin f_{2}(C)$, take $g_{2}=f_{2}$. Otherwise, since all values of $f_{2} \mid C$ are unstable, $\theta$ is unstable, and for the neighborhood $N(\delta)$ there exists a map $g_{2}^{\prime}: C \rightarrow L_{2}^{\prime}$ satisfying $g_{2}^{\prime}(x)=f_{2}(x)$ if $f_{2}(x) \in N(\delta), g_{2}^{\prime}(x) \in N(\delta)$ if $f_{2}(x) \in N(\delta)$, and $\theta \in g_{2}^{\prime}(C)\left[3\right.$, p. 79]. Extend $g_{2}^{\prime} \cup f_{2} \mid S^{n}$ to $g_{2}: E^{n+1}$ $\rightarrow L_{2}$ so that sup $\left\|f_{2}(x)-g_{2}(x)\right\|<\epsilon$. Then $g_{2}$ is the desired map.

Definition (Anderson [2]). A closed subset $A$ of a topological space $X$ has Property $Z$ if for each nonempty homotopically trivial open set $U$ in $X, U \backslash A$ is nonempty and homotopically trivial.

It is readily seen that if $X$ is a space with the property that every open set contains the closure of each of its compact subsets (in particular, if $X$ is Hausdorff or regular), then every homotopically deficient subset of $X$ has Property $Z$.

THEOREM 5.2. Let $X$ be a locally convex linear space and let $A \subset X$ have Property Z. Then $A$ is homotopically deficient.

Proof. Since every convex subset of $X$ is contractible, $A$ must be nowhere dense. Let $f: E^{n+1} \rightarrow X$ with $f\left(S^{n}\right) \cap A=\varnothing$, and let $V$ be a neighborhood of $\theta$. Let $W$ be a circled convex open neighborhood of $\theta$ such that $W+W \subset V$. With $W_{i}=W+x_{i}$, let $\left\{W_{i}: i=1, \cdots, r\right\}$ be 
a finite cover of $f\left(E^{n+1}\right)$. Choose a triangulation $T$ of $E^{n+1}$ that refines $f^{-1}\left\{W_{i}\right\}$. On each vertex $v$ of $T$ define $g(v)=f(v)$ if $f(v) \cap A=\varnothing$, otherwise choose $g(v) \in \cap\left\{W_{i}: f(v) \in W_{i}\right\} \backslash A$. Assume $g$ has been defined on the $k$-skeleton $T^{k}$ of $T$, such that $g\left|T_{j}^{k}=f\right| T_{j}^{k}$ if $f\left(T_{j}^{k}\right) \cap A=\varnothing$, $g\left(T_{j}^{k}\right) \subset \cap\left\{W_{i}: f\left(T_{j}^{k}\right) \subset W_{i}\right\} \backslash A$ for each face $T_{j}^{k}$ of $T^{k}$. Extend $g$ to $T^{k+1}$ as follows: on each face $T_{j}^{k+1}$, let $g\left|T_{j}^{k+1}=f\right| T_{j}^{k+1}$ if $f\left(T_{j}^{k+1}\right) \cap A$ $=\varnothing$, otherwise extend $g \mid \operatorname{Bd}\left(T_{j}^{k+1}\right)$ to $T_{j}^{k+1}$ such that $g\left(T_{j}^{k+1}\right)$ $\subset \cap\left\{W_{i}: f\left(T_{j}^{k+1}\right) \subset W_{i}\right\} \backslash A$ (here we use Property $Z$ and the fact that $\bigcap\left\{W_{i}: f\left(T_{j}^{k+1}\right) \subset W_{i}\right\}$ is convex, hence homotopically trivial). By induction we obtain a map $g: E^{n+1} \rightarrow X$ such that $f\left|S^{n}=g\right| S^{n}$, $(f-g)\left(E^{n+1}\right) \subset V$, and $g\left(E^{n+1}\right) \cap A=\varnothing$.

Homotopy deficiency may be obtained directly for closed totally bounded sets in a situation more general than that indicated by Theorems 3.4 and 5.1:

THEOREM 5.3. Every closed totally bounded subset $A$ of an infinitedimensional Hausdorff linear space $X$ is homotopically deficient.

Proof. By Lemma 2.3, $A$ is nowhere dense. Let $f: E^{n+1} \rightarrow X$ with $f\left(S^{n}\right) \cap A=\varnothing$, and let $V$ be a neighborhood of $\theta$. By compactness there exists a circled neighborhood $W$ of $\theta$ such that $W \subset V$ and $\left(W+W+f\left(S^{n}\right)\right) \cap A=\varnothing$. Since $B=f\left(E^{n+1}\right) \cap(W+A)-A$ is totally bounded, there exists $p \in W \backslash B$. Then $f\left(E^{n+1}\right) \cap(A+p)=\varnothing$. Since $f^{-1}\left(W+f\left(S^{n}\right)\right) \cap f^{-1}(W+A)=\varnothing$, there exists a map $m: E^{n+1} \rightarrow[\theta: p]$ $\subset W$ with $m\left(S^{n}\right)=\theta$ and $m\left(f^{-1}(W+A)\right)=p$. Then $g=f-m$ satisfies $f\left|S^{n}=g\right| S^{n},(f-g)\left(E^{n+1}\right) \subset V$, and $g\left(E^{n+1}\right) \cap A=\varnothing$.

The infinite-dimensionality is obviously necessary; in fact, no nonempty subset of a Euclidean space is homotopically deficient.

\section{BiBLIOGRAPHY}

1. R. D. Anderson, Topological properties of the Hilbert cube and the infinite product of open intervals, Trans. Amer. Math. Soc. 126 (1967), 200-216.

2. - On topological infinite deficiency, Michigan Math. J. 14 (1967), 365-383.

3. W. Hurewicz and H. Wallman, Dimension theory, rev. ed., Princeton Univ. Press, Princeton, N. J., 1948.

4. J. L. Kelley and I. Namioka, Linear topological spaces, Van Nostrand, Princeton, N. J., 1963.

5. V. L. Klee, Convex bodies and periodic homeomorphisms in Hilbert space, Trans. Amer. Math. Soc. 74 (1953), 10-43.

6. - Some topological properties of convex sets, Trans. Amer. Math. Soc. 78 (1955), 30-45.

7. - On the Borelian and projective types of linear subspaces, Math. Scand. 6 (1958), 189-199.

\section{Louisiana State University}

